\title{
Polynomials of Least Deviation from Zero in Sobolev p-Norm
}

\author{
Abel Díaz-González ${ }^{1} \cdot$ Héctor Pijeira-Cabrera ${ }^{1}$ (D) Javier Quintero-Roba ${ }^{1}$
}

Received: 10 July 2021 / Revised: 16 December 2021 / Accepted: 20 December 2021 /

Published online: 13 January 2022

(c) The Author(s) 2022

\begin{abstract}
The first part of this paper complements previous results on characterization of polynomials of least deviation from zero in Sobolev $p$-norm $(1<p<\infty)$ for the case $p=1$. Some relevant examples are indicated. The second part deals with the location of zeros of polynomials of least deviation in discrete Sobolev $p$-norm. The asymptotic distribution of zeros is established on general conditions. Under some order restriction in the discrete part, we prove that the $n$-th polynomial of least deviation has at least $n-\mathbf{d}^{*}$ zeros on the convex hull of the support of the measure, where $\mathbf{d}^{*}$ denotes the number of terms in the discrete part.
\end{abstract}

Keywords Polynomials of least deviation from zero - Extremal polynomials · Sobolev norm $\cdot$ Zero location

Mathematics Subject Classification 30C10 - 30C15 - 33C47 - 41A10 - 41A50 . $41 \mathrm{~A} 52 \cdot 46 \mathrm{E} 35$

\section{Introduction}

Let $\mathbb{P}$ be the linear space of polynomials, $\|\cdot\|$ be a norm defined on $\mathbb{P}$ and $\mathbb{P}_{n}^{1}$ be the subset of all polynomials of degree $n \in \mathbb{Z}_{+}$whose leading coefficient is equal to one

Communicated by Rosihan M. Ali.

Héctor Pijeira-Cabrera hpijeira@math.uc3m.es

Abel Díaz-González

abdiazgo@math.uc3m.es

Javier Quintero-Roba

jaquinte@math.uc3m.es

1 Universidad Carlos III de Madrid, Getafe, Spain 
(monic). A classic problem in analysis is the existence, uniqueness and characterization of the monic polynomial of degree $n \in \mathbb{Z}_{+}$with minimum deviation from zero with respect to the norm $\|\cdot\|$, i.e., the polynomial $P_{n}(z)=z^{n}+\ldots$ such that

$$
\left\|P_{n}\right\|=\inf _{Q_{n} \in \mathbb{P}_{n}^{1}}\left\|Q_{n}\right\|
$$

A polynomial $P_{n} \in \mathbb{P}_{n}^{1}$ that satisfies (1) is called polynomial of least deviation from zero with respect to $\|\cdot\|$, for brevity, a $n$-th minimal (or extremal) polynomial with respect to $\|\cdot\|$. This problem has its origin in the study carried out by P. L. Chebyshev on the decrease in the friction in the joints of the Watt parallelogram that converts the movement of the piston of the steam engine into wheel rotation. As a consequence, what we know today as Chebyshev polynomials were discovered (c.f. [3, Ch. 1]). It is well-known that Chebyshev monic polynomials of the first kind are minimal with respect to the uniform norm at $[-1,1]$ and that those of the second kind are minimal with respect to the usual norm at $L^{1}[-1,1]$ (c.f. $[5, \S 6.6]$ or $[6, \S 3.3]$ ). Let us mention that these works constituted a starting point of the general theory of orthogonal polynomials. Today, minimal polynomials are of great interest in various areas such as approximation theory, potential theory, optimization of numerical algorithms, and signal processing.

Note that, any polynomial $Q \in \mathbb{P}_{n}^{1}$ could be written as $Q(z)=z^{n}-q(z)$ with $q \in \mathbb{P}_{n-1}$. Let $q_{0}$ be a fixed element of $\mathbb{P}_{n-1}$ and define the associated subset

$$
\mathbb{A}_{n, 0}=\left\{q \in \mathbb{P}_{n-1}:\left\|x^{n}-q\right\| \leq\left\|x^{n}-q_{0}\right\|\right\}
$$

As $\mathbb{A}_{n, 0}$ is a compact subset of $\mathbb{P}_{n-1}$, there exists $q_{1} \in \mathbb{P}_{n}$ such that $\left\|x^{n}-q_{1}\right\| \leq\left\|x^{n}-q\right\|$ for all $q \in \mathbb{P}_{n-1}$, in virtue of the arbitrariness of $q_{0}$. Hence, the existence of a minimal polynomial is guaranteed. However, the uniqueness of the minimal polynomial with respect to (2) is not always ensured, as we will show in some of our case studies.

Nevertheless, it is straightforward to prove that $\mathrm{M}_{n}$ (the set of all monic minimal polynomials with respect to $\|\cdot\|$ of degree $n$ ) is a convex set. Indeed, if $Q_{n}, R_{n} \in \mathbb{M}_{n}$ and $\lambda \in[0,1]$, then $P_{n}(x)=\lambda Q_{n}+(1-\lambda) R_{n}(x)$ is also an element of $\mathbb{M}_{n}$ since

$$
\left\|P_{n}\right\|=\left\|\lambda Q_{n}+(1-\lambda) R_{n}(x)\right\| \leq \lambda\left\|Q_{n}\right\|+(1-\lambda)\left\|R_{n}\right\|=\left\|Q_{n}\right\| .
$$

In this paper, we are interested in the case in which the norm $\|\cdot\|$ is as we define below. Let $1 \leq p<\infty$ and consider the vector of measures $\mu=\left(\mu_{0}, \mu_{1}, \ldots, \mu_{m}\right)$, for $m \in \mathbb{Z}_{+}$, where $\mu_{k}$ is a positive finite Borel measure with supp $\mu_{k} \subset \mathbb{R}$ and $\mathbb{P} \subset L^{1}\left(\mu_{k}\right)$ for $k=0,1, \ldots, m$. Denote by $\Delta_{k}$ the convex hull of supp $\mu_{k}$, that is, the smallest interval containing $\operatorname{supp} \mu_{k}$. Let $f^{(k)}$ denote the $k$-th derivative of a function $f$. If $\Delta_{0}$ contains infinite elements, the expression 


$$
\|f\|_{p, \mu}=\left(\sum_{k=0}^{m}\left\|f^{(k)}\right\|_{k, p}^{p}\right)^{1 / p}=\left(\sum_{k=0}^{m} \int_{\Delta_{k}}\left|f^{(k)}\right|^{p} \mathrm{~d} \mu_{k}\right)^{1 / p}
$$

defines a norm over $\mathbb{P}$ known as the Sobolev $p$-norm and the vector of measures $\mu$ is called standard. If each measure $\mu_{k}, 0 \leq k \leq m$ satisfies $\mu_{k}(\{x\})=0$ for all $x \in \mathbb{R}$, we say that the vector of measures $\mu$ is continuous.

First, observe that for $m=0$, this norm reduces to the usual $L^{p}\left(\mu_{0}\right)$ norm. We will call $n$-th Sobolev minimal polynomial with respect to $\|\cdot\|_{p, \mu}$, to any polynomial $P_{n} \in \mathbb{P}_{n}^{1}$ that is a solution of the minimal problem (1).

For the norm (2) with $\mu$ standard, we consider two different cases:

Continuous Sobolev norms, if $\mu$ is continuous.

Discrete Sobolev norms, if for every $k=1, \ldots, m$ the measure $\mu_{k}$ is supported on a finite number of points.

It is said that a Sobolev $p$-norm is sequentially dominated if supp $\mu_{k} \subset \operatorname{supp} \mu_{k-1}$ and $\mathrm{d} \mu_{k}=f_{k-1} \mathrm{~d} \mu_{k-1}$ where $f_{k-1} \in L_{\infty}\left(\mu_{k-1}\right)$ and $k=1, \ldots, m$. Furthermore, the norm (2) on $\mathbb{P}$ is said to be essentially sequentially dominated, if there exists a sequentially dominated norm that is equivalent to (2). As usual, two norms $\|\cdot\|_{1}$ and $\|\cdot\|_{2}$ on a given normed space $\mathbb{X}$ are said to be equivalent if there exist positive constants $c_{1}, c_{2}$ such that $c_{1}\|x\| \leq\|x\| \leq c_{2}\|x\|$ for all $x \in \mathbb{X}$.

The notions of sequentially dominated norm and essentially sequentially dominated norm were introduced in $[15,20]$, respectively. Both notions are closely related to the uniform boundedness of the distance between the zeros of sequences of minimal polynomials and the support of the measures involved in (2). For more details on this aspect in the continuous case, we refer the reader to $[11,16]$ for $p=2$, [17,18] for $1<p<\infty$ and $[8,9,13]$ for $p=2$ and measures with unbounded support.

Let $N \in \mathbb{Z}_{+}, \Omega=\left\{c_{1}, \ldots, c_{N}\right\} \subset \mathbb{C},\left\{m_{0}, \ldots, m_{N}\right\} \subset \mathbb{Z}_{+}$and $m=$ $\max \left\{m_{0}, \ldots, m_{N}\right\}$. In the discrete case, we will restrict our attention to Sobolev $p$ norm under the following assumptions:

- $\mu_{0}=\mu+\sum_{j=1}^{N} A_{j, 0} \delta_{c_{j}}$, where $A_{j, 0} \geq 0, \mu$ is a finite positive Borel measure, supp $\mu \subset \mathbb{R}$ with infinitely many points, $\mathbb{P} \subset L^{1}(\mu)$ and $\delta_{x}$ denotes the Dirac measure with mass one at the point $x$.

- For $k=1, \ldots, m ; \mu_{k}=\sum_{j=1}^{N} A_{j, k} \delta_{c_{j}}$ where $A_{j, k} \geq 0, A_{j, m_{j}}>0$, and $A_{j, k}=0$ if $m_{j}<k \leq m$.

We say that a discrete Sobolev $p$-norm is non-lacunary if $A_{j, k}>0$ for all $0 \leq$ $k \leq m_{j}$ and $0 \leq j \leq N$. In any other case, we say that the discrete Sobolev $p$-norm is lacunary. Obviously, a discrete Sobolev $p$-norm is non-lacunary if and only if is sequentially dominated. A discrete Sobolev $p$-norm is essentially non-lacunary if it is equivalent to a non-lacunary norm.

It is known that the minimal polynomial in $L^{p}\left(\mu_{0}\right)$ spaces $(m=0)$ satisfies the following characterization (see [4, Sec.2.2 and Ex 7-h]). A monic polynomial $P_{n}$ is 
the $n$-th minimal polynomial in $L_{p}\left(\mu_{0}\right)$ if and only if

$$
\begin{gathered}
\left\langle P_{n}, q\right\rangle_{p, \mu_{0}}=\int_{\Delta_{0}} q \operatorname{sgn}\left(P_{n}\right)\left|P_{n}\right|^{p-1} d \mu_{0}=0 \text { for all } q \in \mathbb{P}_{n-1}, \\
\text { where } \operatorname{sgn}(y)= \begin{cases}y /|y|, & \text { if } y \neq 0 \\
0, & \text { if } y=0 .\end{cases}
\end{gathered}
$$

In [10, Th.4], the authors provide the following extension of this characterization to the Sobolev case when $1<p<\infty$.

Theorem 1 Consider the Sobolev p-norm (2) for $1<p<\infty$. Then, the monic polynomial $P_{n}$ is the $n$-th Sobolev minimal polynomial if and only if

$$
\left\langle P_{n}, q\right\rangle_{p, \mu}=\sum_{k=0}^{m} \int_{\Delta_{k}} q^{(k)} \operatorname{sgn}\left(P_{n}^{(k)}\right)\left|P_{n}^{(k)}\right|^{p-1} d \mu_{k}=0,
$$

for every polynomial $q \in \mathbb{P}_{n-1}$.

The results in this work complement previous ones in [10, §2]. There, for $1<p<$ $\infty$, Theorem 1 , Proposition 1 , and Corollary 1 were proved.

The aim of Sect. 2 is to extend Theorem 1 to the case $p=1$. In Theorem 2 , we give a general sufficient condition for existence of a minimal polynomial with respect to (2) $(1 \leq p<\infty)$. For $p=1$, this condition is not necessary, as we show in Examples 2 and 3. Furthermore, Example 1 shows that it does not guarantee uniqueness either. Theorem 3 establishes a necessary and sufficient condition under which (3) characterizes minimality with respect to (2) when $p=1$.

The last two sections deal with discrete Sobolev norms. In Sect. 3, for essentially non-lacunary Sobolev norms, we give a sufficient condition for the uniform boundedness of the set of zeros of a sequence on minimal polynomials $\left\{P_{n}\right\}$ (see Theorem 4). Moreover, the asymptotic distribution of zeros is established in Theorem 5. Finally, in Sect. 4, we introduce the notion of sequentially ordered Sobolev p-norm. Under this assumption, we prove Theorem 7 , which generalizes several known results on the number of zeros of the $n$-th polynomial of least deviation inside the convex hull of the support of the measure $\mu$.

\section{Polynomials of Least Deviation from Zero When $p=1$}

Let us first recall a basic property of the Sobolev norm (2). Let $R$ be a monic polynomial with complex coefficients, and let us write $R=R_{1}+i R_{2}$, where $R_{1}$ and $R_{2}$ are polynomials with real coefficients. Note that $R_{1}$ is also a monic polynomial with the same degree of $R$ and satisfying 


$$
\begin{aligned}
\|R\|_{p, \mu}^{p} & =\sum_{k=0}^{m} \int_{\Delta_{k}}\left|R_{1}^{(k)}+i R_{2}^{(k)}\right|^{p} \mathrm{~d} \mu_{k}=\sum_{k=0}^{m} \int_{\Delta_{k}}\left(\left(R_{1}^{(k)}\right)^{2}+\left(R_{2}^{(k)}\right)^{2}\right)^{p / 2} \mathrm{~d} \mu_{k} \\
& >\sum_{k=0}^{m} \int_{\Delta_{k}}\left|R_{1}^{(k)}\right|^{p} \mathrm{~d} \mu_{k}=\left\|R_{1}\right\|_{p, \mu}^{p} .
\end{aligned}
$$

Therefore, any $n$-th Sobolev minimal polynomial with respect to $\|\cdot\|_{p, \mu}$ has real coefficients.

Proposition 1 ([10, Prop. 1]) Let $\|\cdot\|_{p, \mu}$ be the Sobolev-type norm defined by (2), with $1<p<\infty$. Then, there exists a unique $P_{n} \in \mathbb{P}_{n}^{1}$ such that $\left\|P_{n}\right\|_{p, \mu}=$ $\inf _{Q_{n} \in \mathbb{P}_{n}^{1}}\left\|Q_{n}\right\|_{p, \mu}$.

Theorem 2 (Sufficient condition) Consider the Sobolev $p$-norm (2) for $1 \leq p<\infty$, when $\mu=\left(\mu_{0}, \ldots, \mu_{m}\right)$ is a standard vector measure. If $P_{n} \in \mathbb{P}_{n}^{1}$ is such that for all $q \in \mathbb{P}_{n-1}$

$$
\left\langle P_{n}, q\right\rangle_{p, \mu}=\sum_{k=0}^{m} \int_{\Delta_{k}} q^{(k)}(x) \operatorname{sgn}\left(P_{n}^{(k)}(x)\right)\left|P_{n}^{(k)}(x)\right|^{p-1} d \mu_{k}(x)=0,
$$

then $P_{n}$ is a minimal polynomial with respect to $\|\cdot\|_{p, \mu}$.

Proof If $1<p<\infty$ the proof is carried out as the proof of the sufficiency in [10, Th. 4], step by step.

Hence, in what follows, we consider $p=1$. Write $P_{n}(z)=z^{n}-q_{0}(z)$ where $q_{0} \in \mathbb{P}_{n-1}$, let $q \in \mathbb{P}_{n-1}$ arbitrary and assume that (4) holds, then

$$
\begin{aligned}
\left\|P_{n}\right\|_{1, \mu} & =\sum_{k=0}^{m} \int_{\Delta_{k}}\left(\left(x^{n}\right)^{(k)}-q_{0}^{(k)}(x)\right) \operatorname{sgn}\left(P_{n}^{(k)}(x)\right) \mathrm{d} \mu_{k}(x)=\left\langle P_{n}, x^{n}-q_{0}\right\rangle_{1, \mu} \\
& =\left\langle P_{n}, x^{n}-q+q-q_{0}\right\rangle_{1, \mu}=\left\langle P_{n}, x^{n}-q\right\rangle_{1, \mu}+\left\langle P_{n}, q-q_{0}\right\rangle_{1, \mu} \\
& =\left\langle P_{n}, x^{n}-q\right\rangle_{1, \mu}
\end{aligned}
$$

and taking absolute value, we have

$$
\left\|P_{n}\right\|_{1, \mu} \leq \sum_{k=0}^{m} \int_{\Delta_{k}}\left|\left(x^{n}-q\right)^{(k)}\right| \mathrm{d} \mu_{k}=\left\|x^{n}-q\right\|_{1, \mu}, \quad \forall q \in \mathbb{P}_{n-1},
$$

which is equivalent to the assertion of the theorem for $p=1$.

In [10, Th. 4], it was proved that if $1<p<\infty$, condition (4) is also necessary, i.e., Theorem 2 is a characterization of the extremality in this case.

With the same arguments as in [10, Cor. 1 and Cor. 2], we have the following corollary. 
Corollary 1 Under the assumptions of Theorem 2, if $P_{n} \in \mathbb{P}_{n}^{1}$ satisfies condition (4), then

1. For all $n \geq 1, P_{n}$ has at least one zero of odd multiplicity on $\mathbf{C o}\left(\operatorname{supp} \mu_{0}\right)^{\mathrm{o}}$.

2. For all $n \geq 2, P_{n}^{\prime}$ has at least one zero of odd multiplicity on $\mathbf{C o}\left(\operatorname{supp} \mu_{0} \cup \operatorname{supp} \mu_{1}\right)^{\mathrm{o}}$.

where $\operatorname{Co}(A)$ and $A^{\circ}$ denote the convex hull and the interior of a set $A$, respectively.

Observe that if $p=1$, the condition (4) only depends on the sign of $P_{n}$ and its derivatives on the support of the corresponding measure and not on the values of the polynomial itself. Consequently, unlike what happens in the case $1<p<\infty$, if $p=1$, we lose the uniqueness of the minimal polynomial, as can be seen in the following examples. Furthermore, in Example 2, we obtain a minimal polynomial that does not satisfy the condition (4).

Example 1 (Continuous case)

Consider the Sobolev norm associated with the vector of measures $\mu=$ $\left(\left.v\right|_{[-2,0]},\left.v\right|_{[0,1]}\right)$, where $\left.v\right|_{[a, b]}$ denotes the Lebesgue measure over the real interval $[a . b]$,

$$
\|f\|_{1, \mu}=\int_{-2}^{0}|f| \mathrm{d} x+\int_{0}^{1}\left|f^{\prime}\right| \mathrm{d} x .
$$

Let $P_{a, 2}(x)=(x+1)(x-a)$, with $a \in[0,1]$, a family of monic polynomials of degree 2. Note that

$$
\begin{aligned}
\left\langle P_{a, 2}, 1\right\rangle_{1, \mu} & =\int_{-2}^{0} \operatorname{sgn}((x+1)(x-a)) \mathrm{d} x=\int_{-2}^{-1} \mathrm{~d} x-\int_{-1}^{0} \mathrm{~d} x=0 . \\
\left\langle P_{a, 2}, x\right\rangle_{1, \mu} & =\int_{-2}^{0} x \operatorname{sgn}((x+1)(x-a)) \mathrm{d} x+\int_{0}^{1} \operatorname{sgn}(2 x+1-a) \mathrm{d} x \\
& =\int_{-2}^{-1} x \mathrm{~d} x-\int_{-1}^{0} x \mathrm{~d} x+\int_{0}^{1} \mathrm{~d} x=0 .
\end{aligned}
$$

Then, from Theorem 2, the polynomials $P_{a, 2}$ with $0 \leq a \leq 1$ are all minimal with respect to (5).

Furthermore, note that the minimal polynomials $P_{a, 2}(x)=(x+1)(x-a)$ for all $0 \leq a \leq 1$ are the convex combinations of the minimal polynomials $x^{2}-1$ and $x^{2}+x$.

Example 2 (Discrete case)

Consider the Sobolev norm associated with $\mu=\left(\left.\nu\right|_{[-2,0]}, \delta_{0}\right)$, where $\delta_{0}$ is the Dirac measure with mass one at $x=0$,

$$
\|f\|_{1, \mu}=\int_{-2}^{0}|f| \mathrm{d} x+\left|f^{\prime}(0)\right| .
$$


Let $P_{b, 2}(x)=(x+1)(x-b)$, with $b \in[0,1)$, a family of monic polynomials of degree 2. Note that

$$
\begin{aligned}
\left\langle P_{b, 2}, 1\right\rangle_{1, \mu} & =\int_{-2}^{0} \operatorname{sgn}((x+1)(x-b)) \mathrm{d} x=\int_{-2}^{-1} \mathrm{~d} x-\int_{-1}^{0} \mathrm{~d} x=0 . \\
\left\langle P_{b, 2}, x\right\rangle_{1, \mu} & =\int_{-2}^{0} x \operatorname{sgn}((x+1)(x-b)) \mathrm{d} x+1 \cdot \operatorname{sgn}\left(P_{b, 2}^{\prime}(0)\right) \\
& =\int_{-2}^{-1} x \mathrm{~d} x-\int_{-1}^{0} x \mathrm{~d} x+\operatorname{sgn}(1-b)=0 .
\end{aligned}
$$

Then, from Theorem 2, the polynomials $P_{b, 2}$ with $0 \leq b<1$ are all minimal with respect to (6) and $\left\|P_{b, 2}\right\|_{1, \mu}=2$.

Furthermore, if $b=1$, the polynomials $P_{1,2}(x)=x^{2}-1$ is minimal and does not satisfy condition (4). Indeed,

$$
\begin{aligned}
\left\|P_{1,2}\right\|_{1, \mu} & =2=\left\|P_{b, 2}\right\|_{1, \mu} \text { when } 0 \leq b<1 . \\
\left\langle P_{1,2}, x\right\rangle_{1, \mu} & =\int_{-2}^{0} x \operatorname{sgn}\left(x^{2}-1\right) \mathrm{d} x=-1 \neq 0 .
\end{aligned}
$$

If $1<p<\infty$, from [10, Th. 4], we know that all minimal polynomials with respect to (2) (continuous or discrete case) satisfy the condition (4). But as seen in Example 2 , this statement is not true when $p=1$. It can even happen that there is no minimal polynomial satisfying (4).

\section{Example 3}

Consider the following discrete Sobolev norm,

$$
\|f(x)\|_{1, \mu}=\int_{-1}^{1}|f(x)| \mathrm{d} x+\left|f^{\prime}(0)\right| .
$$

Then, $P_{3}(x)=x^{3}$ is the only third minimal Sobolev polynomial with respect to $\|\cdot\|_{1, \mu}$ and does not satisfy the sufficient condition (4).

1. Note that for every polynomial $Q_{n}$, we have

$$
\left\|(-1)^{n} Q_{n}(-x)\right\|_{1, \mu}=\int_{-1}^{1}\left|Q_{n}(-x)\right| \mathrm{d} x+\left|Q_{n}^{\prime}(0)\right|=\left\|Q_{n}\right\|_{1, \mu} .
$$

2. Then, if $S_{n}$ is a minimal polynomial of degree $n$, the monic polynomial $(-1)^{n} S_{n}(-x)$ is also extremal. From the convexity of the set of minimal polynomials,

$$
P_{n}(x)=\frac{1}{2} S_{n}(x)+\frac{(-1)^{n}}{2} S_{n}(-x)
$$

is an odd or even polynomial, according to the parity of $n$, and a monic minimal polynomial too. 
3. For $n=3$, let $P_{3}(x)=x^{3}+c x$ where $c \in \mathbb{R}$ a monic odd polynomial and

$$
F(c)=\left\|x^{3}+c x\right\|_{1, \mu}=\int_{-1}^{1}\left|x^{3}+c x\right| \mathrm{d} x+|c|= \begin{cases}-2 c-\frac{1}{2}, & c \leq-1 \\ c^{2}+\frac{1}{2}, & -1<c<0 \\ 2 c+\frac{1}{2}, & 0 \leq c\end{cases}
$$

It is straightforward to see that the global minimum of $F$ is attained at $c=0$. Therefore, $P_{3}(x)=x^{3}$ is a minimal polynomial.

4. The polynomial $P_{3}(x)=x^{3}$ does not satisfy (4). Indeed,

$$
\left\langle P_{3}, x\right\rangle_{1, \mu}=\int_{-1}^{1} x \operatorname{sgn}\left(x^{3}\right) \mathrm{d} x=\int_{-1}^{1}|x| \mathrm{d} x=1 \neq 0 .
$$

5. Finally, we will prove the uniqueness. As $P_{3} \in \mathbb{P}_{3}^{1}$ is the only odd minimal polynomial of degree 3 , and that any minimal Sobolev polynomial $S_{3} \in \mathbb{P}_{3}^{1}$ is such that

$$
x^{3}=\frac{1}{2} S_{3}(x)-\frac{1}{2} S_{3}(-x) .
$$

Since $\left\|x^{3}\right\|_{1, \mu}=\frac{1}{2}\left\|S_{3}\right\|_{1, \mu}+\frac{1}{2}\left\|-S_{3}(-x)\right\|_{1, \mu}$, we get

$$
0 \geq \int_{-1}^{1}\left(\left|x^{3}\right|-\frac{1}{2}\left|S_{3}(x)\right|-\frac{1}{2}\left|S_{3}(-x)\right|\right) \mathrm{d} x=\left|S_{3}^{\prime}(0)\right| \geq 0
$$

which implies that $\left|x^{3}\right|=\frac{1}{2}\left|S_{3}(x)\right|+\frac{1}{2}\left|S_{3}(-x)\right|$ and $\left|S_{3}^{\prime}(0)\right|=0$. Consequently, $S_{3}(0)=S_{3}^{\prime}(0)=0$ and $S_{3}$ takes the form $S_{3}(x)=x^{3}+c x^{2}$, with $c \in \mathbb{R}$. Since $c \neq 0$, we arrive at the contradiction

$$
\left\|S_{3}\right\|_{1, \mu}=\int_{-1}^{1}\left|x^{3}+c x^{2}\right| \mathrm{d} x=\left\{\begin{array}{ll}
\frac{1}{2}+\frac{1}{6} c^{4}, & |c|<1 ; \\
\frac{2}{3}|c|, & |c| \geq 1
\end{array}>\frac{1}{2}=\left\|x^{3}\right\|_{1, \mu} .\right.
$$

So, $P_{3}(x)=x^{3}$ is the only minimal Sobolev polynomial of degree 3 .

Note that in this example, we have obtained the only monic minimal polynomial of degree 3 with respect to (7), and it does not satisfy the sufficient condition. This is exclusive to the discrete case. If the vector measure $\mu$ is continuous, the sufficient condition (4) is also necessary.

Theorem 3 Let $\mu=\left(\mu_{0}, \mu_{1}, \ldots, \mu_{m}\right)$ be a continuous standard vector measure. Then, $P_{n}$ is an $n$-th Sobolev minimal polynomial with respect to $\|\cdot\|_{1, \mu}$ if and only if

$$
\left\langle P_{n}, q\right\rangle_{1, \mu}=\sum_{k=0}^{m} \int_{\Delta_{k}} q^{(k)} \operatorname{sgn}\left(P_{n}^{(k)}\right) \mathrm{d} \mu_{k}=0, \quad \forall q \in \mathbb{P}_{n-1} .
$$


Proof From Theorem 2, it only remains to prove that the condition (8) is necessary for the extremality. Without loss of generality, we can assume that $\operatorname{deg} P_{n} \geq m$, since if $n<m$ we have

$$
\left\|P_{n}\right\|_{1, \mu}=\sum_{k=0}^{n} \int_{\Delta_{k}}\left|P_{n}^{(k)}\right| \mathrm{d} \mu_{k}, \text { and the proof works the same. }
$$

Suppose that $P_{n} \in \mathbb{P}_{n}^{1}$ is a minimal polynomial with respect to $\|\cdot\|_{1, \mu}$ and (8) does not hold. Then there exists $h \in \mathbb{P}_{n-1}$ such that $\left\langle P_{n}, h\right\rangle_{1, \mu} \neq 0$. Multiplying $h$ by a constant, we can assume $\left\langle P_{n}, h\right\rangle_{1, \mu}>0$, without loss of generality.

Let $x_{k, 1}<x_{k, 2}<\cdots<x_{k, n_{k}}$ be the zeros of $P_{n}^{(k)}$ which lie on $\Delta_{k}^{o}=\left(a_{k}, b_{k}\right)$. For each $\ell \in \mathbb{N}$ and $k=0, \ldots, m$, denote

$$
A_{k, \ell}=\left[a_{k}+\frac{1}{\ell}, x_{k, 1}-\frac{1}{\ell}\right] \cup\left[x_{k, 1}+\frac{1}{\ell}, x_{k, 2}-\frac{1}{\ell}\right] \cup \cdots \cup\left[x_{k, n_{k}}+\frac{1}{\ell}, b_{k}-\frac{1}{\ell}\right] .
$$

Note that $\left\{A_{k, \ell}\right\}_{\ell}$ is a sequence of compact subsets of $\Delta_{k}^{o}$, such that $A_{k}:=\lim _{\ell \rightarrow \infty} A_{k, \ell}=$ $\Delta_{k}^{\mathrm{o}} \backslash \Lambda_{k}$, where $\Lambda_{k}=\left\{x_{k, 1}, x_{k, 2}, \ldots, x_{k, n_{k}}\right\}$. Let $B_{k, \ell}=\Delta_{k}^{\mathrm{o}} \backslash A_{k, \ell}$, so $\lim _{\ell \rightarrow \infty} B_{k, \ell}=\Lambda_{k}$.

As $\mu$ is a vector of continuous measures, for every $k=0,1, \ldots, m$, we have

$$
\begin{gathered}
\lim _{\ell \rightarrow \infty} \int_{A_{k, \ell}} h^{(k)} \operatorname{sgn}\left(P_{n}^{(k)}\right) \mathrm{d} \mu_{k}=\int_{A_{k}} h^{(k)} \operatorname{sgn}\left(P_{n}^{(k)}\right) \mathrm{d} \mu_{k}=\int_{\Delta_{k}} h^{(k)} \operatorname{sgn}\left(P_{n}^{(k)}\right) \mathrm{d} \mu_{k}, \\
\lim _{\ell \rightarrow \infty} \int_{B_{k, \ell}}\left|h^{(k)}\right| \mathrm{d} \mu_{k}=\int_{\Lambda_{k}}\left|h^{(k)}\right| \mathrm{d} \mu_{k}=0 .
\end{gathered}
$$

Therefore,

$$
\begin{aligned}
\lim _{\ell \rightarrow \infty} \sum_{k=0}^{m} \int_{A_{k, \ell}} h^{(k)} \operatorname{sgn}\left(P_{n}^{(k)}\right) \mathrm{d} \mu_{k} & =\left\langle P_{n}, h\right\rangle_{1, \mu}>0, \\
\lim _{\ell \rightarrow \infty} \sum_{k=0}^{m} \int_{B_{k, \ell}}\left|h^{(k)}\right| \mathrm{d} \mu_{k} & =0 .
\end{aligned}
$$

Hence, for $\ell_{0} \in \mathbb{N}$ sufficiently large,

$$
\sum_{k=0}^{m} \int_{A_{k, \ell_{0}}} h^{(k)} \operatorname{sgn}\left(P_{n}^{(k)}\right) \mathrm{d} \mu_{k}>\sum_{k=0}^{m} \int_{B_{k, \ell_{0}}}\left|h^{(k)}\right| \mathrm{d} \mu_{k} \cdot
$$

Since every set $A_{k, \ell_{0}}, k=0,1, \ldots, m$ is compact and $\Lambda_{k} \cap A_{k, \ell_{0}}=\emptyset$, we get

$$
\delta=\min _{k=0,1, \ldots, m}\left\{\min _{x \in A_{k, \ell_{0}}}\left\{\left|P_{n}^{(k)}(x)\right|\right\}\right\}>0 .
$$


From the compactness of $A_{k, \ell_{0}}$, we also obtain that

$$
\delta_{h}=\max _{k=0,1, \ldots, m}\left\{\max _{x \in A_{k, \ell_{0}}}\left\{\left|h^{(k)}(x)\right|\right\}\right\}
$$

is finite and positive. Then, we can choose $\lambda>0$ such that $0<\lambda<\frac{\delta}{\delta_{h}}$.

Therefore, for each $k=0,1, \ldots, m$, we have $\left|\lambda h^{(k)}(x)\right|<\delta \leq\left|P_{n}^{(k)}(x)\right|$ for all $x \in A_{k, \ell_{0}}$ and

$$
\operatorname{sgn}\left(P_{n}^{(k)}(x)-\lambda h^{(k)}(x)\right)=\operatorname{sgn}\left(P_{n}^{(k)}(x)\right), \quad \text { for all } x \in A_{k, \ell_{0}} .
$$

Finally,

$$
\begin{aligned}
\left\|P_{n}-\lambda h\right\|_{1, \mu}= & \sum_{k=0}^{m} \int_{\Delta_{k}}\left|P_{n}^{(k)}-\lambda h^{(k)}\right| \mathrm{d} \mu_{k} \\
= & \sum_{k=0}^{m}\left(\int_{B_{k, \ell_{0}}}\left|P_{n}^{(k)}-\lambda h^{(k)}\right| \mathrm{d} \mu_{k}+\int_{A_{k, \ell_{0}}}\left|P_{n}^{(k)}-\lambda h^{(k)}\right| \mathrm{d} \mu_{k}\right) \\
= & \sum_{k=0}^{m}\left(\int_{B_{k, \ell_{0}}}\left|P_{n}^{(k)}-\lambda h^{(k)}\right| \mathrm{d} \mu_{k}+\int_{A_{k, \ell_{0}}} \operatorname{sgn}\left(P_{n}^{(k)}-\lambda h^{(k)}\right)\left(P_{n}^{(k)}-\lambda h^{(k)}\right) \mathrm{d} \mu_{k}\right) \\
= & \sum_{k=0}^{m}\left(\int_{B_{k, \ell_{0}}}\left|P_{n}^{(k)}-\lambda h^{(k)}\right| \mathrm{d} \mu_{k}+\int_{A_{k, \ell_{0}}} \operatorname{sgn}\left(P_{n}^{(k)}\right)\left(P_{n}^{(k)}-\lambda h^{(k)}\right) \mathrm{d} \mu_{k}\right) \\
\leq & \sum_{k=0}^{m}\left(\int_{B_{k, \ell_{0}}}\left|P_{n}^{(k)}\right| \mathrm{d} \mu_{k}+\lambda \int_{B_{k, \ell_{0}}}\left|h^{(k)}\right| \mathrm{d} \mu_{k}\right. \\
& \left.+\int_{A_{k, \ell_{0}}}\left|P_{n}^{(k)}\right| \mathrm{d} \mu_{k}-\lambda \int_{A_{k, \ell_{0}}} \operatorname{sgn}\left(P_{n}^{(k)}\right) h^{(k)} \mathrm{d} \mu_{k}\right) \\
= & \sum_{k=0}^{m} \int_{\Delta_{k}}\left|P_{n}^{(k)}\right| \mathrm{d} \mu_{k}+\lambda\left(\sum_{k=0}^{m} \int_{B_{k, \ell_{0}}}\left|h^{(k)}\right| \mathrm{d} \mu_{k}-\sum_{k=0}^{m} \int_{A_{k, \ell_{0}}} \operatorname{sgn}\left(P_{n}^{(k)}\right) h^{(k)} \mathrm{d} \mu_{k}\right) \\
< & \left\|P_{n}\right\|_{1, \mu},
\end{aligned}
$$

which is a contradiction with the extremality of $P_{n}$.

\section{Lacunary and Non-lacunary Discrete Sobolev Norms}

Most of the formulas given here are known to the specialist, although precise references may be hard to find in the literature. Therefore, we include this section with full proofs for completeness, except when an exact reference is available.

Consider a finite positive Borel measure $\mu$, being supp $\mu$ a subset of the real line with infinitely many points such that $\mathbb{P} \subset L^{1}(\mu)$. In the remainder, we 
assume that $N \in \mathbb{Z}_{+}, \Omega=\left\{c_{1}, c_{2}, \ldots, c_{N}\right\} \subset \mathbb{R},\left\{m_{0}, m_{1}, \ldots, m_{N}\right\} \subset \mathbb{Z}_{+}$and $m=\max \left\{m_{0}, m_{1}, \ldots, m_{N}\right\}$. Let $\mu=\left(\mu_{0}, \mu_{1}, \ldots, \mu_{m}\right)$ be the standard vector measure. For each $1 \leq p<\infty$, let us consider the general discrete Sobolev norm

$$
\|f\|_{p, \mu}=\left(\sum_{k=0}^{m} \int_{\Delta_{k}}\left|f^{(k)}\right|^{p} \mathrm{~d} \mu_{k}\right)^{1 / p}=\left(\int_{\Delta}|f|^{p} \mathrm{~d} \mu+\sum_{j=1}^{N} \sum_{k=0}^{m_{j}} A_{j, k}\left|f^{(k)}\left(c_{j}\right)\right|^{p}\right)^{1 / p},
$$

where $\Delta$ is the convex hull of the support of the measure $\mu$. Notice that unlike (2), the representation (9) of $\|\cdot\|_{p, \mu}$ is not unique, but depends on how many Dirac measures, of the discrete part of $\mu_{0}$, are included in the measure $\mu$. In general, the representation (9) is unique once the measure $\mu$ is fixed, so this dependence will be omitted for brevity.

If there exists a constant $M$ such that

$$
\|x q\|_{p, \mu} \leq M\|q\|_{p, \mu}, \quad \text { for all } q \in \mathbb{P}
$$

we say that the multiplication operator is bounded on $\mathbb{P}$ with respect to $\|\cdot\|_{p, \mu}$. The close relation between (10) and the uniform boundedness of the set of zeros of sequences of minimal polynomials was established in [15]. Since then, several studies have been published on this subject.

Proposition 2 Assume that the discrete Sobolev norm (9) is non-lacunary and $\Delta$ is bounded, then for each $q \in \mathbb{P}$, we have

$$
\begin{aligned}
& \|x q\|_{p, \mu} \leq M\|q\|_{p, \mu}, \\
& \text { where } M=\max \left\{M_{1}, 2^{p-1}\left(M_{1}+m M_{2}\right)\right\}^{1 / p}, M_{1}=\sup _{x \in K}|x|^{p}, K=\Delta \cup\left\{c_{1}, \ldots, c_{m}\right\}, \\
& M_{2}=\max \left\{\frac{A_{j, k+1}}{A_{j, k}}: 1 \leq j \leq N \text { and } 0 \leq k \leq m_{j}-1\right\}
\end{aligned}
$$

Proof Notice that $(x q)^{(k)}=x q^{(k)}+k q^{(k-1)}, \quad k \in \mathbb{N}$. Therefore,

$$
\begin{aligned}
\Psi & :=\sum_{j=1}^{N} \sum_{k=0}^{m_{j}} A_{j, k}\left|c_{j} q^{(k)}\left(c_{j}\right)+k q^{(k-1)}\left(c_{j}\right)\right|^{p} \\
& \leq 2^{p-1}\left(\sum_{j=1}^{N} \sum_{k=0}^{m_{j}} A_{j, k}\left|c_{j} q^{(k)}\left(c_{j}\right)\right|^{p}+\sum_{j=1}^{N} \sum_{k=1}^{m_{j}} A_{j, k}\left|k q^{(k-1)}\left(c_{j}\right)\right|^{p}\right) \\
& \leq 2^{p-1}\left(M_{1} \sum_{j=1}^{N} \sum_{k=0}^{m_{j}} A_{j, k}\left|q^{(k)}\left(c_{j}\right)\right|^{p}+m \sum_{j=1}^{N} \sum_{k=1}^{m_{j}} A_{j, k}\left|q^{(k-1)}\left(c_{j}\right)\right|^{p}\right)
\end{aligned}
$$




$$
\begin{aligned}
& =2^{p-1}\left(M_{1} \sum_{j=1}^{N} \sum_{k=0}^{m_{j}} A_{j, k}\left|q^{(k)}\left(c_{j}\right)\right|^{p}+m \sum_{j=1}^{N} \sum_{k=0}^{m_{j}-1} A_{j, k+1}\left|q^{(k)}\left(c_{j}\right)\right|^{p}\right) \\
& \leq 2^{p-1}\left(M_{1} \sum_{j=1}^{N} \sum_{k=0}^{m_{j}} A_{j, k}\left|q^{(k)}\left(c_{j}\right)\right|^{p}+m M_{2} \sum_{j=1}^{N} \sum_{k=0}^{m_{j}-1} A_{j, k}\left|q^{(k)}\left(c_{j}\right)\right|^{p}\right) \\
& \leq 2^{p-1}\left(\left(M_{1}+m M_{2}\right) \sum_{j=1}^{N} \sum_{k=0}^{m_{j}} A_{j, k}\left|q^{(k)}\left(c_{j}\right)\right|^{p}\right) \cdot \\
\|x q\|_{p, \mu}^{p} & =\int_{\Delta}|x q|^{p} d \mu+\Psi \\
& \leq M_{1} \int_{\Delta}|q|^{p} \mathrm{~d} \mu+2^{p-1}\left(\left(M_{1}+m M_{2}\right) \sum_{j=1}^{N} \sum_{k=0}^{m_{j}} A_{j, k}\left|q^{(k)}\left(c_{j}\right)\right|^{p}\right) \\
& \leq M^{p}\|q\|_{p, \mu}^{p} .
\end{aligned}
$$

If $\|\cdot\|_{p, \mu}$ is a lacunary Sobolev norm defined as in (9), we define the associated non-lacunary norm as $\|\cdot\|_{p, \mu^{*}}$

$$
\|f\|_{p, \mu^{*}}=\left(\int_{\Delta}|f|^{p} \mathrm{~d} \mu+\sum_{j=1}^{N} \sum_{k=0}^{m_{j}} A_{j, k}^{*}\left|f^{(k)}\left(c_{j}\right)\right|^{p}\right)^{1 / p}
$$

where $A_{j, k}^{*}= \begin{cases}A_{j, k}, & \text { if } A_{j, k}>0 \text { or } m_{j}<k \leq m \\ 1, & \text { in other case. }\end{cases}$

Proposition 3 Let $\|\cdot\|_{p, \mu}$ be a lacunary Sobolev norm defined as in (9), with $\Delta$ bounded. Then, there exists a constant $M$ such that $\|x q\|_{p, \mu} \leq M\|q\|_{p, \mu}$ for all $q \in \mathbb{P}$ if and only if the lacunary norm (9) and the associated non-lacunary norm (11) are equivalents (i.e., $\|\cdot\|_{p, \mu}$ is essentially non-lacunary).

Proof Assume that a lacunary norm defined as in (9) is equivalent to its associated non-lacunary norm (11). From Proposition 2, it is straightforward that there exists a constant $M$ such that $\|x q\|_{p, \mu} \leq M\|q\|_{p, \mu}$.

Now, suppose that the multiplication operator is bounded on $\mathbb{P}$ with respect to the lacunary norm $\|\cdot\|_{p, \mu}$, then there exist $M>0:\|x q\|_{p, \mu} \leq\|q\|_{p, \mu}, q \in \mathbb{P}$. From (11), obviously $\|q\|_{p, \mu} \leq\|q\|_{p, \mu^{*}}$. Furthermore, from definition

$$
\|q\|_{p, \mu^{*}}=\left(\|q\|_{p, \mu}^{p}+\sum_{j=1}^{N} \sum_{k \in I_{j}}\left|q^{(k)}\left(c_{j}\right)\right|^{p}\right)^{1 / p} \leq\|q\|_{p, \mu}+\left(\sum_{j=1}^{N} \sum_{k \in I_{j}}\left|q^{(k)}\left(c_{j}\right)\right|^{p}\right)^{1 / p}
$$


where $I_{j}=\left\{k: A_{j, k}=0\right.$ and $\left.0 \leq k<m_{j}\right\}$. Therefore, the remainder of the proof is devoted to find a constant $K^{*}$ such that

$$
\left(\sum_{j=1}^{N} \sum_{k \in I_{j}}\left|q^{(k)}\left(c_{j}\right)\right|^{p}\right)^{1 / p} \leq K^{*}\|q\|_{p, \mu} \quad q \in \mathbb{P} .
$$

To achieve this purpose, it is sufficient to prove that for every $j$ and $0 \leq k<m_{j}$, there exists a constant $K_{j, k}>0$ satisfying

$$
\left|q^{(k)}\left(c_{j}\right)\right| \leq K_{j, k}\|q\|_{p, \mu} \quad q \in \mathbb{P}
$$

In this case, taking $K^{*}=\left(\sum_{j=1}^{N} \sum_{k \in I_{j}} K_{j, k}^{p}\right)^{1 / p}$, we get (12).

To prove the inequality (13), note that

$$
\begin{aligned}
\left|(k+1) q^{(k)}\left(c_{j}\right)\right|-\left|c_{j} q^{(k+1)}\left(c_{j}\right)\right| & \leq\left|(k+1) q^{(k)}\left(c_{j}\right)+c_{j} q^{(k+1)}\left(c_{j}\right)\right|=\left|(x q)^{(k+1)}\left(c_{j}\right)\right|, \\
\left|q^{(k)}\left(c_{j}\right)\right| \leq\left|(k+1) q^{(k)}\left(c_{j}\right)\right| & \leq\left|(x q)^{(k+1)}\left(c_{j}\right)\right|+\left|c_{j} q^{(k+1)}\left(c_{j}\right)\right| \\
& \leq\left|(x q)^{(k+1)}\left(c_{j}\right)\right|+\left|c^{*}\right|\left|q^{(k+1)}\left(c_{j}\right)\right|,
\end{aligned}
$$

where $c^{*}=\max _{1 \leq j \leq N}\left|c_{j}\right|$. If $m_{j}-k=1$, and $q \in \mathbb{P}$

$$
\begin{aligned}
\left|q^{\left(m_{j}-1\right)}\left(c_{j}\right)\right| & \leq \frac{1}{A_{j, m_{j}}}\left|A_{j, m_{j}}(x q)^{\left(m_{j}\right)}\left(c_{j}\right)\right|+\frac{\left|c^{*}\right|}{A_{j, m_{j}}}\left|A_{j, m_{j}} q^{\left(m_{j}\right)}\left(c_{j}\right)\right| . \\
& \leq \frac{1}{A_{j, m_{j}}}\|x q\|_{p, \mu}+\frac{\left|c^{*}\right|}{A_{j, m_{j}}}\|q\|_{p, \mu} \leq K_{j, m_{j}-1}\|q\|_{p, \mu} .
\end{aligned}
$$

where $K_{j, m_{j}-1}=\frac{M+\left|c^{*}\right|}{A_{j, m_{j}}} \neq 0$ and we get (13) for $k=m_{j}-1$.

We now proceed by induction.

1. $\left[m_{j}-k=\ell\right]$ Assume that (13) holds for $k=m_{j}-\ell$, i.e., there exists a constant $K_{j, m_{j}-\ell} \neq 0$ such that

$$
\left|q^{\left(m_{j}-\ell\right)}\left(c_{j}\right)\right| \leq K_{j, m_{j}-\ell}\|q\|_{p, \mu} .
$$

2. [ $\left.m_{j}-k=\ell+1\right]$ If $k=m_{j}-\ell-1$, from (14) and the induction hypothesis

$$
\begin{aligned}
\left|q^{\left(m_{j}-\ell-1\right)}\left(c_{j}\right)\right| & \leq\left|(x q)^{\left(m_{j}-\ell\right)}\left(c_{j}\right)\right|+\left|c^{*}\right|\left|q^{\left(m_{j}-\ell\right)}\left(c_{j}\right)\right| \\
& \leq K_{j, m_{j}-\ell}\|x q\|_{p, \mu}+K_{j, m_{j}-\ell}\left|c^{*}\right|\|q\|_{p, \mu} \leq K_{j, m_{j}-\ell-1}\|q\|_{p, \mu}
\end{aligned}
$$


where $K_{j, m_{j}-\ell-1}=\left(M+\left|c^{*}\right|\right) K_{j, m_{j}-\ell}$.

Theorem 4 If (9) is essentially non-lacunary, then the set of zeros of a minimal polynomial sequence is uniformly bounded.

Proof Let (9) be an essentially non-lacunary Sobolev norm and (11) its associated nonlacunary Sobolev norm. From Proposition 3, there exist constants $C_{1}, C_{2}>0$ such that $C_{1}\|q\|_{p, \mu^{*}} \leq\|q\|_{p, \mu} \leq C_{2}\|q\|_{p, \mu^{*}}$ for all $q \in \mathbb{P}$. Moreover, from Proposition 2 , there exists another constant $C_{3}>0$ such that $\|z q\|_{p, \mu^{*}} \leq C_{3}\|q\|_{p, \mu^{*}}$.

If $P_{n}$ is a minimal polynomial of degree $n$ and $P_{n}\left(z_{0}\right)=0$, there exists a monic polynomial $q$ of degree $n-1$ such that $P_{n}(z)=\left(z-z_{0}\right) q(z)$. As $P_{n}$ is minimal

$$
\left|z_{0}\right|\|q\|_{p, \mu}-\|z q\|_{p, \mu} \leq\left\|z_{0} q-z q\right\|_{p, \mu}=\left\|P_{n}\right\|_{p, \mu} \leq\|z q\|_{p, \mu}
$$

Then,

$$
\left|z_{0}\right| C_{1}\|q\|_{p, \mu^{*}} \leq\left|z_{0}\right|\|q\|_{p, \mu} \leq 2\|z q\|_{p, \mu} \leq 2 C_{2}\|z q\|_{p, \mu^{*}} \leq 2 C_{2} C_{3}\|q\|_{p, \mu^{*}},
$$

which completes the proof.

\subsection{Asymptotic Distribution of Zeros}

To state the result on the zero distribution of minimal polynomials with respect to an essentially non-lacunary norm, we need to introduce some concepts and notations.

- For any polynomial $q$ of exact degree $n$, we denote $\vartheta(q)=\frac{1}{n} \sum_{j=1}^{n} \delta_{z_{j}}$, where $z_{1}, \ldots, z_{n}$ are the zeros of $q$ repeated according to their multiplicity. This is the so called normalized counting measure associated with $q$.

- If $\Delta=\operatorname{supp} \mu$ is regular (a compact subset of the complex plane is said to be regular if the unbounded connected component of its complement is regular with respect to the Dirichlet problem), the measure $\mu \in \mathbf{R e g}$ if and only if

$$
\lim _{n \rightarrow \infty}\left(\frac{\left\|q_{n}\right\|_{\Delta}}{\left\|q_{n}\right\|_{p, \mu}}\right)^{1 / n}=1,
$$

for every sequence of polynomials $\left\{q_{n}\right\}, \operatorname{deg} q_{n} \leq n, q_{n} \not \equiv 0$ (cf. [21, Th 3.4.3]), where $\|\cdot\|_{\mathscr{A}}$ denotes the supremum norm on $\mathscr{A} \subset \mathbb{C}$.

- Given a compact set $\mathscr{A} \subset \mathbb{C}, \operatorname{cap}(\mathscr{A})$ denotes the logarithmic capacity of $\mathscr{A}, \omega_{\mathscr{A}}$ the equilibrium measure on $\mathscr{A}$ and $G_{\mathscr{A}}(z ; \infty)$ the corresponding Green's function with singularity at infinity (cf. [19,21]).

- Let $T_{n}$ be the $n$-th monic minimal polynomial with respect to $\|\cdot\|_{\Delta}$, i.e., the $n$-th Chebyshev polynomial with respecto to $\Delta$. It is known that

$$
\lim _{n \rightarrow \infty}\left\|T_{n}\right\|_{\Delta}^{1 / n}=\operatorname{cap}(\Delta) . \quad[19, \text { Cor. 5.5.5] }
$$


To determine the asymptotic distribution of zeros of sequences of minimal polynomials in this section, we need the following lemma.

Lemma 1 [15, Lemma 3] Let E be a compact regular subset of the complex plane and $\left\{q_{n}\right\}$ a sequence of polynomials such that $\operatorname{deg} q_{n} \leq n$ and $q_{n} \not \equiv 0$. Then, for all $k \in \mathbb{Z}_{+}$,

$$
\varlimsup_{n \rightarrow \infty} \sqrt[n]{\frac{\left\|q_{n}^{(k)}\right\|_{E}}{\left\|q_{n}\right\|_{E}}} \leq 1 .
$$

The following theorem is the main result of this section and is valid for discrete Sobolev norms, whether lacunary or not. For $p=2$, the theorem was proved in $[15$, Th. 5], and for continuous Sobolev norms in [17, Th. 2]. The scheme of the proof is quite similar to the previous ones.

Theorem 5 Consider a discrete Sobolev p-norm (9), such that $\mu \in \operatorname{Reg}$ and $\Delta$ is a bounded real interval. If $\left\{P_{n}\right\}$ is the sequence of monic minimal polynomials with respect to (9), then for all $j \geq 0$

$$
\begin{aligned}
\lim _{n \rightarrow \infty}\left\|P_{n}^{(j)}\right\|_{\Delta}^{1 / n} & =\operatorname{cap}(\Delta), \text { and } \\
\mathrm{W}_{n \rightarrow \infty} \vartheta\left(P_{n}^{(j)}\right) & =\omega_{\Delta}, \quad \text { in the weak topology of measures. }
\end{aligned}
$$

Proof Firstly, the compact set $\Delta$ has empty interior and connected complement, and under these conditions (see [2, Th. 2.1]), we have that (18) implies (19).

Let $T_{n}$ be the $n$-th monic minimal polynomial with respect to $\|\cdot\|_{\Delta}$, i.e., the $n$-th Chebyshev polynomial with respecto to $\Delta$. From (16), it is straightforward to see that for all sequence $\left\{Q_{n}\right\}_{n \in \mathbb{Z}_{+}}$of monic polynomials $Q_{n}$ of degree $n$

$$
\begin{gathered}
\underline{\lim }_{n \rightarrow \infty}\left\|Q_{n}^{(j)}\right\|_{\Delta}^{1 / n} \geq \underline{\lim }_{n \rightarrow \infty}\left\|T_{n-j}\right\|_{\Delta}^{1 / n}=\operatorname{cap}(\Delta) \\
\text { If } \rho(z)=\prod_{j=1}^{N}\left(z-c_{j}\right)^{m_{j}+1} \text { and } n \geq \mathbf{d}:=N+\sum_{j=1}^{N} m_{j} \text {, we get } \\
\left\|P_{n}\right\|_{p, \mu}^{p} \leq\left\|P_{n}\right\|_{p, \mu}^{p} \leq\left\|\rho T_{n-\mathbf{d}}\right\|_{p, \mu}^{p}=\int_{\Delta}\left|\rho T_{n-\mathbf{d}}\right|^{p} \mathrm{~d} \mu \leq \mu(\Delta)\|\rho\|_{\Delta}^{p}\left\|T_{n-\mathbf{d}}\right\|_{\Delta}^{p} .
\end{gathered}
$$

From (15)-(16), $\varlimsup_{n \rightarrow \infty}\left\|P_{n}\right\|_{\Delta}^{1 / n} \leq \operatorname{cap}(\Delta)$. Therefore, as $\Delta$ is a compact regular set, from (17), we have for every $j \geq 0$

$$
\varlimsup_{n \rightarrow \infty}\left\|P_{n}^{(j)}\right\|_{\Delta}^{1 / n} \leq \operatorname{cap}(\Delta)
$$

Finally, from (20)-(21), we get (18). 
If the norm (9) is essentially non-lacunary, from Theorem 4, we know that there exists a constant $M$ such that

$$
\left\{z \in \mathbb{C}: P_{n}(z)=0 \text { for some } n \in \mathbb{Z}_{+}\right\} \subset D_{M}=\{z \in \mathbb{C}:|z| \leq M\}
$$

where $\left\{P_{n}\right\}$ is a sequence of minimal polynomials with respect to (9) $\left(\operatorname{deg}\left(P_{n}\right)=n\right)$. Under this consideration, we have the following asymptotic results.

Corollary 2 Assume that $\left\{P_{n}\right\}$ is the sequence of minimal polynomials with respect to an essentially non-lacunary norm (9), where $\Delta$ is regular and $\mu \in \mathbf{R e g}$. Then, for all $j \in \mathbf{Z}_{+}$

1. $\varlimsup_{n \rightarrow \infty}\left|P_{n}^{(j)}(z)\right|^{1 / n}=\operatorname{cap}(\Delta) e^{G_{\Delta}(z ; \infty)}$, for every $z \in \mathbb{C}$ except for a set of capacity zero,

2. $\lim _{n \rightarrow \infty}\left|P_{n}^{(j)}(z)\right|^{1 / n}=\operatorname{cap}(\Delta) e^{G_{\Delta}(z ; \infty)}$, uniformly on compact subsets of $\Omega=$ $\mathbb{C} \backslash D_{M}$.

3. $\lim _{n \rightarrow \infty} \frac{P_{n}^{(j+1)}(z)}{n P_{n}^{(j)}(z)}=\int_{\Delta} \frac{d \omega_{\Delta}(x)}{z-x}$, uniformly on compact subsets of $\Omega$.

Proof From Proposition 3, it is sufficient to prove the corollary for non-lacunary norms. As it was commented for the case $p=2$ in the last paragraph of [15], the proof here follows [17, Th. 6] point by point to get the desired result.

\section{Sequentially Ordered Discrete Sobolev Norm}

If the discrete Sobolev norm (9) is non-lacunary, it is easy to prove that the $n$-th minimal Sobolev polynomial has all its the zeros located on $\Delta$, except a number of them equal to the amount of nonzero values $A_{j, k}$ in the discrete part of (9); see Proposition 4. In this section, we extend this result to lacunary Sobolev norms when the discrete part of (9) satisfies certain order condition.

Fix $1<p<\infty$ and a standard vector measure $\mu$ such that $\|\cdot\|_{p, \mu}$ is a discrete Sobolev norm defined by (9) and satisfying $c_{j} \notin \Delta^{\mathrm{o}}=(a, b)$ for $j=1,2, \ldots, N$. As in the previous section, consider the polynomial

$$
\rho(x)=\prod_{c_{j} \leq a}\left(x-c_{j}\right)^{m_{j}+1} \prod_{c_{j} \geq b}\left(c_{j}-x\right)^{m_{j}+1}
$$

of degree $\mathbf{d}=N+\sum_{j=1}^{N} m_{j}$ and positive on $(a, b)$. If $n>\mathbf{d}$ and $P_{n}$ is the $n$-th minimal polynomial with respect to (9), from Theorem 1

$$
\int_{a}^{b} q \operatorname{sgn}\left(P_{n}\right)\left|P_{n}\right|^{p-1} \rho \mathrm{d} \mu=\left\langle P_{n}, q \rho\right\rangle_{p, \mu}=0
$$


for every $q \in \mathbb{P}_{n-\mathbf{d}-1}$. Hence, the polynomial $P_{n}$ has at least $n-\mathbf{d}$ changes of sign on $\Delta^{\mathrm{o}}$, otherwise (22) lead us to a contradiction with

$$
\int_{a}^{b} q \operatorname{sgn}\left(P_{n}\right)\left|P_{n}\right|^{p-1} \rho \mathrm{d} \mu>0,
$$

where $q$ is the polynomial having a simple zero on each change of sign of $P_{n}$ on $(a, b)$. So, we have proved the following proposition, which is the extension of [12, Proposition 2.1] to the minimal case, $1<p<\infty$.

Proposition 4 Let $P_{n}$ be the $n$-th Sobolev minimal polynomial with respect to (9) $(1<p<\infty)$, which satisfies $c_{j} \notin \Delta^{\circ}$ for $j=1,2, \ldots, N$, and $n>\mathbf{d}$, then $P_{n}$ has at least $(n-\mathbf{d})$ changes of sign on $\Delta^{\mathrm{o}}$.

Proposition 4 can also be seen as a generalization of the zero location theorem for standard orthogonal polynomials ( $p=2$ and $m=0$ ). However, a result proved by M. G. Bruin already in 1993, see [7, Th. 4.1], seems to suggest that the number of zeros of $P_{n}$ in $\Delta^{\mathrm{o}}$ does not depend only on the higher-order derivatives $m_{j}$ of each point $c_{j}$, but on the number of terms in the discrete part of (9)

$$
\mathbf{d}^{*}:=\left|\left\{A_{j, k}>0: j=1,2, \ldots, N, k=0,1, \ldots, m_{j}\right\}\right|,
$$

where $|A|$ denotes the cardinality of a set $A$.

This assumption became even stronger when the relative asymptotic of discrete Sobolev orthogonal polynomials [14, Theorem 4] was found. Finally, in [1], the authors proved it for the case when (9) has only one mass point $(N=1)$.

Theorem 6 ([1, Th. 2.2]) Let $\mu$ be a standard measure such that $c \in \mathbb{R} \backslash \Delta^{\mathrm{o}}$. If $P_{n}$ denotes the $n$-th Sobolev minimal polynomial with respect to

$$
\|f\|_{2, \mu}=\left(\int_{\Delta}|f|^{2} d \mu+\sum_{k=0}^{m} A_{k}\left|f^{(k)}(c)\right|^{2}\right)^{1 / 2},
$$

then $P_{n}$ has at least $n-\mathbf{d}^{*}$ changes of sign in $\Delta^{\mathrm{o}}$.

The next examples show that this theorem is not longer true if we consider arbitrary mass point configurations with more than one point (i.e., $N \geq 2$ in (9)), at least not for every value of $n$.

Example 4 (bounded case) Set

$$
\|f\|_{2, \mu}=\left(\int_{-1}^{1}|f|^{2} \mathrm{~d} x+8\left|f^{\prime}(4)\right|^{2}+6\left|f^{\prime \prime}(2)\right|^{2}\right)^{1 / 2}
$$

then

$$
P_{4}(x)=k_{4}\left(x^{4}-\frac{2595}{803} x^{3}-\frac{5232}{539} x^{2}-\frac{837735}{39347} x+\frac{8181}{2695}\right)
$$


whose zeros are approximately $\xi_{1} \approx 0.13, \xi_{2} \approx-5.62, \xi_{3} \approx-1.26+1.56 i$ and $\xi_{4} \approx-1.26-1.56 i$.

Example 5 (unbounded case) Set

$$
\|f\|_{2, \mu}=\left(\int_{0}^{\infty}|f(x)|^{2} e^{-x} \mathrm{~d} x+3\left|f^{\prime}(-4)\right|^{2}+8\left|f^{\prime \prime}(0)\right|^{2}\right)^{1 / 2}
$$

then

$$
P_{4}(x)=k_{4}\left(x^{4}-\frac{128}{97} x^{3}-\frac{2536}{97} x^{2}+\frac{8800}{97} x-\frac{5288}{97}\right)
$$

whose zeros are approximately $\xi_{1} \approx 0.78, \xi_{2} \approx-5.93, \xi_{3} \approx 3.24+1.16 i$ and $\xi_{4} \approx 3.24-1.16 i$.

Note that in both cases, three zeros of $P_{4}$ are out of $\Delta^{\circ}$ and two of them are non-real. The first result treating the case $N \geq 2$ in a general way is [12, Theorem 1]. Here, the authors give a result similar to Theorem 6 for $N \geq 2$ in the case $p=2$, and the discrete part of (9) satisfies certain order condition. The condition was called by the authors the sequentially order condition. Although the condition was enough for the purposes of the paper, it does not include the case of Theorem 6, when there is more than one order derivative at the same mass point $c_{j}$. Following the same technique, we expand this condition a little bit more, in such a way that the case of Theorem 6 is included. We will remain calling it the sequentially order condition or we will simply say that the discrete Sobolev norm is sequentially ordered. The result is also generalized for the minimal case $1<p<\infty$.

Definition 1 (Sequentially ordered Sobolev norm) We say that a discrete Sobolev norm $\|\cdot\|_{p, \mu}$ defined by (9) is sequentially ordered if the conditions

$$
\Delta_{k} \cap \mathbf{C o}\left(\cup_{i=0}^{k-1} \Delta_{i}\right)^{\mathrm{o}}=\varnothing, \quad k=1,2, \ldots, m, \text { hold }
$$

We recall that $\Delta_{k}:=\mathbf{C o}\left(\operatorname{supp} \mu_{k}\right)$, so in the discrete case they can be rewritten as

$$
\Delta_{k}= \begin{cases}\operatorname{Co}\left(\Delta \cup\left\{c_{j}: A_{j, 0}>0\right\}\right), & \text { if } k=0 \\ \mathbf{C o}\left(\left\{c_{j}: A_{j, k}>0\right\}\right), & \text { if } 1 \leq k \leq m\end{cases}
$$

Example 6 The following Sobolev discrete norms are sequentially ordered for any $p \in[1, \infty)$ and a standard measure $\mu$ 


$$
\begin{aligned}
\|f\|_{p, \mu} & =\left(\int_{-1}^{1}|f|^{p} \mathrm{~d} \mu+4\left|f^{\prime}(-1)\right|^{p}+\left|f^{\prime}(-3)\right|^{p}+3\left|f^{\prime \prime}(2)\right|^{p}+5\left|f^{(5)}(-3)\right|^{p}\right)^{1 / p} . \\
\|f\|_{p, \mu} & =\left(\int_{-1}^{1}|f|^{p} \mathrm{~d} \mu+\sum_{k=0}^{\ell_{1}} A_{1, k}\left|f^{(k)}(-1)\right|^{p}+\sum_{k=0}^{\ell_{2}} A_{2, k}\left|f^{(k)}(1)\right|^{p}\right)^{1 / p} .
\end{aligned}
$$

where $A_{1, k} A_{2, k}=0$ for $k=0,1, \ldots, \min \left\{\ell_{1}, \ell_{2}\right\}$.

Theorem 7 Let $\mu$ be a standard vector measure and $1<p<\infty$. If $\|\cdot\|_{p, \mu}$ is a sequentially ordered Sobolev norm written as (9), where $\mu$ is taken in such a way $c_{j} \notin \Delta^{\mathrm{o}}$, then $P_{n}$ has at least $n-\mathbf{d}^{*}$ changes of sign on $\Delta^{\mathrm{o}}$.

It is worth noting that although the theorem is enunciated depending on which representation (9) of the Sobolev norm is considered, the definition of sequentially ordered Sobolev norm is independent of this representation. If what we are after is to locate the largest possible number of zeros, we should calculate $\mathbf{d}^{*}$ in the theorem considering the representation (2), rather than (9). However, in this case, we would have the zeros located in the bigger set $\Delta_{0} \supset \Delta$. Because of the assumption $c_{j} \notin \Delta^{\mathrm{o}}$, this inclusion is strict except for the trivial case of (2) and (9) agree $\left(\mu \equiv \mu_{0}\right)$.

Notice that both Examples 4 and 5 are not sequentially ordered. So, this order restriction in the discrete part seems to be optimal to have the most number of zeros simple and located on $\Delta^{\mathrm{o}}$, at least for every value of $n$.

\subsection{Proof of Theorem 7}

Given a polynomial $Q$ with real coefficients and a real set $A$, we introduce the following notations:

- $\mathbf{N}_{o}(Q ; A)$ denotes the number of values on $A$ where the polynomial $Q$ vanishes, (i.e., zeros of $Q$ on $A$ without counting multiplicities).

- $\mathbf{N}_{z}(Q ; A)$ denotes the total number of zeros (counting multiplicities) of $Q$ on $A$.

The next lemma is an extension of [16, Lem. 2.1] and [12, Lem. 3.1].

Lemma 2 Let $\left\{I_{k}\right\}_{k=0}^{m}$ be a set of intervals on the real line with $m \in \mathbb{Z}_{+}$and let $Q$ be a polynomial with real coefficients of degree $\geq m$. If

$$
I_{k} \cap \mathbf{C o}\left(\cup_{i=0}^{k-1} I_{i}\right)^{\mathrm{o}}=\emptyset, \quad k=1,2, \ldots, m,
$$

then

$$
\begin{aligned}
& \mathbf{N}_{z}(Q ; J)+\mathbf{N}_{o}\left(Q ; I_{0} \backslash J\right)+\sum_{i=1}^{m} \mathbf{N}_{o}\left(Q^{(i)} ; I_{i}\right) \\
& \quad \leq \mathbf{N}_{z}\left(Q^{(m)} ; J\right)+\mathbf{N}_{o}\left(Q^{(m)} ; \mathbf{C o}\left(\cup_{i=0}^{m} I_{i}\right) \backslash J\right)+m,
\end{aligned}
$$


for every closed subinterval $J$ of $I_{0}^{\mathrm{O}}$ (both empty set and unitary sets are assumed to be intervals).

Proof First, we are going to point out the following consequence of Rolle's Theorem. If $I$ is a real interval and $J$ is a closed subinterval of $I^{\mathrm{o}}$, then

$$
\mathbf{N}_{z}(Q ; J)+\mathbf{N}_{o}(Q ; I \backslash J) \leq \mathbf{N}_{z}\left(Q^{\prime} ; J\right)+\mathbf{N}_{o}\left(Q^{\prime} ; I^{9} J\right)+1
$$

For $m=0$ (24) trivially holds. We now proceed by induction on $m$. Suppose that we have $m+1$ intervals $\left\{I_{i}\right\}_{i=0}^{m}$ satisfying (23), and that (24) is true for the first $m$ intervals $\left\{I_{k}\right\}_{k=0}^{m-1}$. From (25), we obtain

$$
\begin{aligned}
& \mathbf{N}_{z}(Q ; J)+\mathbf{N}_{o}\left(Q ; I_{0} \backslash J\right)+\sum_{i=1}^{m} \mathbf{N}_{o}\left(Q^{(i)} ; I_{i}\right) \\
& \quad \leq \mathbf{N}_{z}\left(Q^{(m-1)} ; J\right)+\mathbf{N}_{o}\left(Q^{(m-1)} ; \mathbf{C o}\left(\cup_{i=0}^{m-1} I_{i}\right) \backslash J\right)+m-1+\mathbf{N}_{o}\left(Q^{(m)} ; I_{m}\right) \\
& \quad \leq \mathbf{N}_{z}\left(Q^{(m)} ; J\right)+\mathbf{N}_{o}\left(Q^{(m)} ; \mathbf{C o}\left(\cup_{i=0}^{m-1} I_{i}\right)^{o} \backslash J\right)+m+\mathbf{N}_{o}\left(Q^{(m)} ; I_{m}\right) \\
& \quad \leq \mathbf{N}_{z}\left(Q^{(m)} ; J\right)+\mathbf{N}_{o}\left(Q^{(m)} ; \mathbf{C o}\left(\cup_{i=0}^{m} I_{i}\right) \backslash J\right)+m
\end{aligned}
$$

Corollary 3 Under the hypotheses of the above lemma, we have

$$
\mathbf{N}_{z}(Q ; J)+\mathbf{N}_{o}\left(Q ; I_{0} \backslash J\right)+\sum_{i=1}^{m} \mathbf{N}_{o}\left(Q^{(i)} ; I_{i}\right) \leq \operatorname{deg} Q
$$

for every $J$ closed subinterval of $I_{0}^{\mathrm{O}}$. In particular for $J=\emptyset$, we get

$$
\sum_{i=0}^{m} \mathbf{N}_{o}\left(Q^{(i)} ; I_{i}\right) \leq \operatorname{deg} Q
$$

Definition 2 We say that a sequence of ordered pairs $\left\{\left(r_{i}, v_{i}\right)\right\}_{i=1}^{M} \subset \mathbb{R} \times \mathbb{Z}_{+}$is sequentially ordered, if $v_{1} \leq v_{2} \leq \cdots \leq v_{M}$ and the set of intervals $I_{k}=\operatorname{Co}\left(\left\{r_{i}: v_{i}=k\right\}\right)$, $k=0,1, \ldots, v_{M}$, satisfy conditions (23).

Lemma 3 Let $\left\{\left(r_{i}, v_{i}\right)\right\}_{i=1}^{M} \subset \mathbb{R} \times \mathbb{Z}_{+}$be a sequence of $M$ ordered pairs, then there exists a unique monic polynomial $U_{M}$ of minimal degree $(\leq M)$, such that

$$
U_{M}^{\left(v_{i}\right)}\left(r_{i}\right)=0, \quad i=1,2, \ldots, M .
$$

Furthermore, if $\left\{\left(r_{i}, v_{i}\right)\right\}_{i=1}^{M}$ is sequentially ordered, then the degree of $U_{M}$ is $\mathfrak{u}_{M}=$ $\min \mathfrak{I}_{M}-1$, where

$$
\mathfrak{I}_{M}=\left\{i: 1 \leq i \leq M \text { and } v_{i} \geq i\right\} \cup\{M+1\}
$$


Proof The existence of a non-identically zero polynomial with degree $\leq M$ satisfying (28) reduces to solving a homogeneous linear system of $M$ equations with $M+1$ unknowns (its coefficients). Thus, a non trivial solution always exists. In addition, if we suppose that there exist two different minimal monic polynomials $U_{M}$ and $\widetilde{U}_{M}$, then the polynomial $\widehat{U}_{M}=U_{M}-\widetilde{U}_{M}$ is not identically zero, it satisfies (28), and $\operatorname{deg} \widehat{U}_{M}<\operatorname{deg} U_{M}$. So, if we divide $\widehat{U}_{M}$ by its leading coefficient, we reach a contradiction.

The rest of the proof runs by induction on the number of points $M$. For $M=1$, the result follows taking

$$
U_{1}(x)= \begin{cases}x-r_{1}, & \text { if } v_{1}=0 \\ 1, & \text { if } v_{1} \geq 1\end{cases}
$$

Suppose that for each sequentially ordered sequence of $M-1$ ordered pairs, the corresponding minimal polynomial $U_{M-1}$ has degree $\mathfrak{u}_{M-1}$.

Let $\left\{\left(r_{i}, v_{i}\right)\right\}_{i=1}^{M}$ be a sequentially ordered sequence of $M$ ordered pairs. Obviously, $\left\{\left(r_{i}, v_{i}\right)\right\}_{i=1}^{M-1}$ is a sequence of $M-1$ ordered pairs which is sequentially ordered, $\operatorname{deg} U_{M} \geq \operatorname{deg} U_{M-1}$, and from the induction hypothesis $\operatorname{deg} U_{M-1}=\mathfrak{u}_{M-1}$. Now, we shall split the proof in two cases:

1. If $\mathfrak{u}_{M}=M$, then for all $1 \leq i \leq M$, we have $v_{i}<i$, which yields

$$
\operatorname{deg} U_{M} \geq \operatorname{deg} U_{M-1}=\mathfrak{u}_{M-1}=M-1 \geq v_{M} .
$$

Since $\left\{\left(r_{i}, v_{i}\right)\right\}_{i=1}^{M}$ is sequentially ordered, from (27), we get

$$
M \leq \sum_{i=0}^{v_{M}} \mathbf{N}_{o}\left(U_{M}^{(i)} ; I_{i}\right) \leq \operatorname{deg} U_{M}
$$

which implies that $\operatorname{deg} U_{M}=M=\mathfrak{u}_{M}$.

2. If $\mathfrak{u}_{M} \leq M-1$, then there exists a minimal $j(1 \leq j \leq M)$, such that $v_{j} \geq j$, and $v_{i}<i$ for all $1 \leq i \leq j-1$. Therefore, $\mathfrak{u}_{M}=j-1=\mathfrak{u}_{M-1}$. From the induction hypothesis,

$$
\operatorname{deg} U_{M-1}=\mathfrak{u}_{M-1}=j-1 \leq v_{j}-1 \leq v_{M}-1,
$$

which gives $U_{M-1}^{\left(v_{M}\right)} \equiv 0$. Hence, $U_{M} \equiv U_{M-1}$ and, consequently, we get

$$
\operatorname{deg} U_{M}=\operatorname{deg} U_{M-1}=\mathfrak{u}_{M-1}=\mathfrak{u}_{M} .
$$

Note that in Lemma 3, the assumption of $\left\{\left(r_{i}, v_{i}\right)\right\}_{i=1}^{M}$ being sequentially ordered is necessary for asserting that the polynomial $U_{M}$ has degree $\mathfrak{u}_{M}$. In fact, if we consider $\{(-1,0),(1,0),(0,1)\}$, which is no sequentially ordered, we get $U_{3}=x^{2}-1$ and $\mathfrak{u}_{3}=3 \neq \operatorname{deg} U_{3}$. 
Proof of Theorem 7 Let $\xi_{1}<\xi_{2}<\cdots<\xi_{\eta}$ be the points on $\Delta^{o}$ where $P_{n}$ changes sign and suppose that $\eta<n-\mathbf{d}^{*}$. Since $\|\cdot\|_{p, \mu}$ is sequentially ordered, the sequence of $\mathbf{d}^{*}+\eta$ ordered pairs

$$
\left\{\left(r_{i}, v_{i}\right)\right\}_{i=1}^{\mathbf{d}^{*}+\eta}=\left\{\left(\xi_{i}, 0\right)\right\}_{i=1}^{\eta} \cup\left\{\left(c_{j}, k\right): A_{j, k}>0, j=1, \ldots, N, k=0, \ldots, m_{j}\right\}
$$

is sequentially ordered. (We can assume without loss of generality that $v_{1} \leq v_{2} \leq$ $\cdots \leq v_{\mathbf{d}^{*}+\eta}$.) Consequently, from Lemma 3, there exists a unique monic polynomial $U_{\mathbf{d}^{*}+\eta}$ of minimal degree, such that

$$
\begin{aligned}
& U_{\mathbf{d}^{*}+\eta}\left(\xi_{i}\right)=0, \quad \text { for } i=1, \ldots, \eta ; \\
& U_{\mathbf{d}^{*}+\eta}^{(k)}\left(c_{j}\right)=0, \quad \text { for }(j, k): A_{j, k}>0 \text {; }
\end{aligned}
$$

and $\operatorname{deg} U_{\mathbf{d}^{*}+\eta}=\min \mathfrak{I}_{\mathbf{d}^{*}+\eta}-1 \leq \mathbf{d}^{*}+\eta$, where

$$
\mathfrak{I}_{\mathbf{d}^{*}+\eta}=\left\{i: 1 \leq i \leq \mathbf{d}^{*}+\eta \text { and } \nu_{i} \geq i\right\} \cup\left\{\mathbf{d}^{*}+\eta+1\right\} .
$$

Now, we need to consider the following two cases.

1. If $\operatorname{deg} U_{\mathbf{d}^{*}+\eta}=\mathbf{d}^{*}+\eta$, from (30), we get $\operatorname{deg} U_{\mathbf{d}^{*}+\eta}=\mathbf{d}^{*}+\eta \geq v_{\eta+\mathbf{d}^{*}}+1$. Thus, taking $I_{i}=\Delta_{i}, i=0,1, \ldots, m$ and the closed interval $J=\left[\xi_{1}, \xi_{\eta}\right] \subset \Delta^{\mathrm{o}} \subset \Delta_{0}^{\mathrm{o}}$ in (26), we get

$$
\begin{aligned}
\mathbf{d}^{*}+\eta \leq & \sum_{k=0}^{v_{\mathbf{d}^{*}+\eta}} \mathbf{N}_{o}\left(U_{\mathbf{d}^{*}+\eta}^{(k)} ; \Delta_{k}\right) \leq \mathbf{N}_{z}\left(U_{\mathbf{d}^{*}+\eta} ; J\right)+\mathbf{N}_{o}\left(U_{\mathbf{d}^{*}+\eta} ; \Delta_{0} \backslash J\right) \\
& +\sum_{k=1}^{v_{\mathbf{d}^{*}+\eta}} \mathbf{N}_{o}\left(U_{\mathbf{d}^{*}+\eta}^{(k)} ; \Delta_{k}\right) \leq \operatorname{deg} U_{\mathbf{d}^{*}+\eta}=\mathbf{d}^{*}+\eta
\end{aligned}
$$

2. If $\operatorname{deg} U_{\mathbf{d}^{*}+\eta}<\mathbf{d}^{*}+\eta$, from (30), there exists $1 \leq j \leq \mathbf{d}^{*}+\eta$ such that $\operatorname{deg} U_{\mathbf{d}^{*}+\eta}=j-1, v_{j} \geq j$ and $v_{i} \leq i-1$ for $i=1,2, \ldots, j-1$. Hence,

$$
v_{j-1}+1 \leq j-1=\operatorname{deg} U_{\mathbf{d}^{*}+\eta}
$$

and, again, from (26), we have

$$
\begin{aligned}
j-1 \leq & \sum_{k=0}^{v_{j-1}} \mathbf{N}_{o}\left(U_{\mathbf{d}^{*}+\eta}^{(k)} ; \Delta_{k}\right) \leq \mathbf{N}_{z}\left(U_{\mathbf{d}^{*}+\eta} ; J\right)+\mathbf{N}_{o}\left(U_{\mathbf{d}^{*}+\eta} ; \Delta_{0} \backslash J\right) \\
& +\sum_{k=1}^{v_{j-1}} \mathbf{N}_{o}\left(U_{\mathbf{d}^{*}+\eta}^{(k)} ; \Delta_{k}\right) \leq \operatorname{deg} U_{\mathbf{d}^{*}+\eta}=j-1 .
\end{aligned}
$$

In both cases, we obtain that $U_{\mathbf{d}^{*}+\eta}$ has no other zeros in $\Delta_{0}$ than those given by construction and from $\mathbf{N}_{o}\left(U_{\mathbf{d}^{*}+\eta} ; J\right)=\mathbf{N}_{z}\left(U_{\mathbf{d}^{*}+\eta} ; J\right)$ we obtain that all the zeros on 
$\Delta^{0}$ are simple. Thus, in addition to (29), we get that $P_{n} U_{\mathbf{d}^{*}+\eta}$ does not change sign on $\Delta^{\mathrm{o}}$. So we have

$$
\begin{aligned}
\left\langle P_{n}, U_{\mathbf{d}^{*}+\eta}\right\rangle_{p, \mu}= & \int_{\Delta} U_{\mathbf{d}^{*}+\eta} \operatorname{sgn}\left(P_{n}\right)\left|P_{n}\right|^{p-1} \mathrm{~d} \mu \\
& +\sum_{j=1}^{N} \sum_{k=0}^{m_{j}} A_{j, k} U_{\mathbf{d}^{*}+\eta}^{(k)}\left(c_{j}\right) \operatorname{sgn}\left(P_{n}^{(k)}\left(c_{j}\right)\right)\left|P_{n}^{(k)}\left(c_{j}\right)\right|^{p-1} \\
= & \int_{\Delta} U_{\mathbf{d}^{*}+\eta} \operatorname{sgn}\left(P_{n}\right)\left|P_{n}\right|^{p-1} \mathrm{~d} \mu \neq 0 .
\end{aligned}
$$

Since $\operatorname{deg} U_{\mathbf{d}^{*}+\eta} \leq \mathbf{d}^{*}+\eta<n$, we arrive at a contradiction with Theorem 1 .

Acknowledgements The research of H. Pijeira-Cabrera was partially supported by Ministry of Science, Innovation and Universities of Spain, under grant PGC2018-096504-B-C33.

Funding Open Access funding provided by Universidad Carlos III de Madrid thanks to the CRUE-CSIC 2021 agreement with Springer Nature.

Open Access This article is licensed under a Creative Commons Attribution 4.0 International License, which permits use, sharing, adaptation, distribution and reproduction in any medium or format, as long as you give appropriate credit to the original author(s) and the source, provide a link to the Creative Commons licence, and indicate if changes were made. The images or other third party material in this article are included in the article's Creative Commons licence, unless indicated otherwise in a credit line to the material. If material is not included in the article's Creative Commons licence and your intended use is not permitted by statutory regulation or exceeds the permitted use, you will need to obtain permission directly from the copyright holder. To view a copy of this licence, visit http://creativecommons.org/licenses/by/4.0/.

\section{References}

1. Alfaro, M., López Lagomasino, G., Rezola, M.L.: Some properties of zeros of Sobolev-type orthogonal polynomials. J. Comput. Appl. Math. 69, 171-179 (1996)

2. Blatt, H.P., Saff, E.B., Simkani, M.: Jentzsch-Szegö type theorems for the zeros of best approximates. J. Lond. Math. Soc. 38, 192-204 (1988)

3. Bogatyrev, A.: Extremal Polynomials and Riemann Surfaces. Springer, Berlin (2012)

4. Borwein, P., Erdelyi, T.: Polynomials and Polynomial Inequalities. Graduate Texts in Mathematics, vol. 161. Springer, New York (1995)

5. Cheney, E.W.: Introduction to Approximation Theory. AMS Chelsea Pub, Providence (1982)

6. Davis, P.J.: Interpolation and Approximation. Dover Publ, New York (1975)

7. de Bruin, M.G.: A tool for locating zeros of orthogonal polynomials in Sobolev inner product spaces. J. Comput. Appl. Math. 49, 27-35 (1993)

8. Díaz, C., Orive, R., Pijeira, H.: Asymptotically extremal polynomials with respect to varying weights and application to Sobolev orthogonality. J. Math. Anal. Appl. 346, 480-488 (2008)

9. Díaz, C., Orive, R., Pijeira, H.: Zeros and logarithmic asymptotics of Sobolev orthogonal polynomials for exponential weights. J. Comput. Appl. Math. 233, 691-698 (2009)

10. Díaz, A., López Lagomasino, G., Pijeira, H.: Asymptotic zero distribution for a class of extremal polynomials. Bull. Math. Sci. 11, 950019-1-950019-18 (2021)

11. Duran, A., Saff, E.: Zero location for nonstandard orthogonal polynomials. J. Approx. Theory 113, 127-141 (2001)

12. Díaz, A., Pijeira, H., Pérez, I.: Rational approximation and Sobolev-type orthogonality. J. Approx. Theory 260, 105481-1-105481-19 (2020) 
13. López Lagomasino, G., Marcellán, F., Pijeira, H.: Logarithmic asymptotics of contracted Sobolev extremal polynomials on the real line. J. Approx. Theory 143, 62-73 (2006)

14. López Lagomasino, G., Marcellán, F., Van Assche, W.: Relative asymptotics for orthogonal polynomials with respect to a discrete Sobolev inner product. Constr. Approx. 11, 107-137 (1995)

15. López Lagomasino, G., Pijeira, H.: Zero location and $n$-th root asymptotics of Sobolev orthogonal polynomials. J. Approx. Theory 99, 30-43 (1999)

16. López Lagomasino, G., Pijeira, H., Pérez, I.: Sobolev orthogonal polynomials in the complex plane. J. Comput. Appl. Math. 127, 219-230 (2001)

17. López Lagomasino, G., Pérez, I., Pijeira, H.: Asymptotics of extremal polynomials in the complex plane. J. Approx. Theory 137, 226-237 (2005)

18. Portilla, A., Quintana, Y., Rodríguez, J.M., Tourís, E.: Concerning asymptotic behavior for extremal polynomials associated to non-diagonal Sobolev norms. J. Funct. Spaces Appl. 2013, 1-13 (2013)

19. Ransford, T.: Potential Theory in the Complex Plane. Cambridge University Press, Cambridge (1995)

20. Rodríguez, J.M.: The multiplication operator in Sobolev spaces with respect to measures. J. Approx. Theory 109, 157-197 (2001)

21. Stahl, H., Totik, V.: General Orthogonal Polynomials. Cambridge Univ. Press, Cambridge (1992)

Publisher's Note Springer Nature remains neutral with regard to jurisdictional claims in published maps and institutional affiliations. 